\title{
ASPECTOS BIOLÓGICOS DO PARASITÓIDE CHELONUS INSULARIS (CRESSON) (HYMENOPTERA, BRACONIDAE) CRIADOS EM OVOS DE SPODOPTERA FRUGIPERDA (SMITH) (LEPIDOPTERA, NOCTUIDAE)
}

\author{
Maria A.A. Rezende ${ }^{1}$ \\ Ivan $\mathrm{Cruz}^{2}$ \\ Terezinha M.C. Della Lucia ${ }^{1}$
}

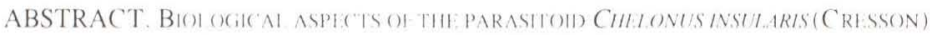

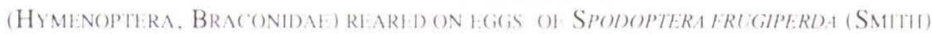
(LiPII)PTtRA. BRACONIDAB.). Biological aspects of Chelontes insularis (Cresson. 1865) an egg-larval parasitoid reared on Spodoptera frugiperda (Smith. 1797) eggs Chelonus insularis (Cresson. 1865) has been cited in the international literature as a promissing biological control agent against the fall armyworm. Spodoptera frugiperda (Smith. 1797), Its field oceurrence in Sete Lagoas. Minas Gerais (Brasil) has been increasing in the last years. For these reasons this experiment was conducted in the laboratory, at the National Corn and Sorghum Research Center. of EMBRAPA. under temperature of $25^{\circ} \mathrm{C}$. RH of $73 \%$ and photophase of 12 hours. Ten couples of the parasitoid were individually placed in a glass jar (5 liters capacity). They were fed on a $10 \%$ sugar solution. Each couple received one fall armyworm egg mass to oviposit during a 24 -hour period. After hatching. the larvae were fed on artificial bean diet. up to the death caused by the parasitoid larvae. The total biological life cycle was 26.61 days on the average (larval period of 20.42 and pupal period of 6.19 days). The average weight of two days - old pupae was (0.) () $g$. The adult longevity was on average. 10 days $(8.4$ days for males and 11.6 days for females). The greatest rate of parasitism occurred when the fenale was three days old. with a maximum of 92 eggs parasitized in that day.

KEY WORDS. Braconidae. Chelonus insularis. Noctuidae. Spodoptera frusiperda. Biological control
\end{abstract}

Várias espécies de Chelonus (Panzer, 1806) são encontradas em diversos paises sendo registradas no Brasil, Argentina, México e Estados Unidos (MARSH et al. 1979; DE SANTIS 1967, 1980), dentre outros.

As fêmeas de Chelonus insularis (Cresson, 1865), de acordo com PIERCE \& Holloway (1912) ovipositam nos ovos do hospedeiro, mas ao invés dos parasitóides emergirem desses, eles emergem como larvas das lagartas imaturas do hospedeiro. A fêmea de $C$. insularis remove a porção de pelos dos aglomerados

1) Departamento de Bioloğa Animal. Universidade Federal de Viçosa. 36570-(0)(x) Viçosa. Minas Gerais. Brasil.

2) Centro Nacional de Pesquisa de Milho e sorgo. Empresa Brasileira de Pesquisa Agropecuária. Caixa Postal 151, 35701-970) Sete Lagoas. Minas Gerais. Brasil. 
dos ovos e coloca seus ovos nos ovos da mariposa (WolcotT 1948). Segundo UlzyetT (1949), C. texanus Cresson (1872) é um parasitóide solitário e arrenótoco, completando três instares no hospedeiro antes de emergir e se desenvolver externamente. O mesmo acontece com os parasitóides do mesmo gênero, $C$. phthorimaeae (Gahan, 1917) e C. kellieae Marsh, 1979 que são primários, solitários, e endoparasitóides ovo-larvais de Phthorimaea operculella (Zeller, 1873) (Lepidoptera, Gelechiidae). A larva do parasitóide deixa a lagarta hospedeira de primeiro instar e continua alimentando-se externamente. Nas duas espécies, os adultos emergem da pupa durante a fase clara do fotoperíodo, sendo que os machos emergem aproximadamente dois dias antes das fêmeas (POWERS \& OATMAN 1984).

O potencial de parasitismo do gênero Chelonus tem sido estudado para diferentes pragas. Por exemplo, a percentagem de parasitismo de Chelonus blackburni (Cameron, 1886) em ovos de Corcyra cephalonica (Stainton, 1865) (Lepidoptera, Galleriidae) e Earias vittella (Fabricius, 1794) (Lepidoptera, Noctuidae), pragas do algodão foi de $72,3 \%$ e $50,6 \%$ respectivamente. Em Pectinophora gossypiella (Saunders, 1844) (Lepidoptera, Gelechiidae) a fêmea de Chelonus colocou 909 ovos, indicando uma alta capacidade reprodutiva quando comparada com outras espécies de microhimenópteros (JACKSON et al. 1978; SWAMIAPPAN \& BALASUBRAMANIAN 1979).

Em C. phthorimaeae, o número total da progênie de fêmeas virgens foi de 449 emergindo somente machos, enquanto das fêmeas acasaladas foi de 568, consistindo de machos e fềmeas. A longevidade de ambas, fêmeas virgens e acasaladas foi de 21,7 dias e 16,5 dias respectivamente. O período reprodutivo abrangeu $86,8 \%$ do período de vida das fêmeas virgens e $90,5 \%$ das fêmeas acasaladas. As fêmeas acasaladas produziram mais adultos por dia do que fêmeas virgens (POWERS \& OATMAN 1984).

Considerando o reduzido volume de informações a respeito de $C$. insularis e a sua possibilidade de utilização num programa de controle biológico, este trabalho teve por finalidade investigar alguns aspectos da biologia deste inseto criado em $S$. frugiperda, bem como seu potencial de parasitismo na lagarta do cartucho do milho.

\section{MATERIAL E MÉTODOS}

O trabalho foi conduzido em laboratório do Centro Nacional de Pesquisa de Milho e Sorgo (CNPMS/EMBRAPA), Sete Lagoas, Minas Gerais. A criação de Chelonus insularis foi estabelecida a partir de adultos coletados no campo e mantidos em gaiolas de posturas em laboratório, sob fotofase de $12 \mathrm{~h}$, a uma temperatura de $25 \pm 2^{\circ} \mathrm{C}$ e $73+2 \%$ de umidade relativa. As lagartas de Spodoptera frugiperda utilizadas como hospedeiros foram criadas em dieta artificial a base de feijão e germe de trigo (PERKINS et al. 1973) sob as mesmas condições de laboratório.

No estudo da biologia do parasitóide, dez casais de $C$. insularis recém emergidos foram individualizados e colocados em vidro transparente de boca larga 
com cinco litros de capacidade, modificado por RECHAV (1978). Cada casal recebeu como alimento uma solução açucarada (um litro de água, 10g de açúcar e um grama de ácido ascórbico), através de um chumaço de algodão embebido, e foi mantido em laboratório sob condições controladas. A cada $24 \mathrm{~h}$ foi oferecida ao parasitóide uma postura de $S$. frugiperda constituída de várias camadas de ovos não contados, sendo esse procedimento efetuado a partir do segundo dia de emergência, diariamente, até a morte das fêmeas do parasitóide.

Após a eclosão, as lagartas hospedeiras foram alimentadas com dieta artificial e após uma semana, foram individualizadas em copos plásticos de $50 \mathrm{ml}$, contendo dieta artificial semelhantemente ao método utilizado por SWAMIAPPAN \& BALASUBRAMANIAN (1979) e POWERS \& OATMAN (1984).

Esses recipientes foram vedados com tampa de acrílico e dispostos em suportes de isopor com 24 divisões e observados diariamente.

Foram avaliados os seguintes parâmetros biológicos: a) período de incubação do parasitóide $C$. insularis, estudado mediante a dissecação diária de cinco ovos e cinco lagartas parasitadas; b) período de pré-oviposição; c) período larval e pupal incluindo peso de 20 pupas com dois dias de idade; d) longevidade do adulto macho e fêmea; e) potencial de parasitismo a partir do segundo dia de emergência das fêmeas, sendo considerados apenas os adultos de $C$. insularis obtidos.

\section{RESULTADOS E DISCUSSÃO}

Verificou-se que não existe período de pré-oviposição para Chelonus insularis e que o período médio de incubação foi de 1,8 dias. Os ovos depositados são de tamanho reduzido; aproximadamente $0,18 \mathrm{~mm}$ de comprimento, com as extremidades arredondadas, semelhantes aos de $C$. inanitus observados por HAFFES et al. (1980) os quais, segundo estes autores, são piriformes. O período larval variou de 17 a 23 dias, apresentando média geral de 20,42 dias e o período pupal apresentou uma média de 6,19 dias (máximo de nove e mínimo de três dias) (Tab. I). A duração média do ciclo total foi de 26,61 dias (máximo de 33 e mínimo de 26 dias). O peso médio de 20 pupas com dois dias foi de $0,02 \mathrm{~g}$.

Tabela I. Parâmetros biológicos de Chelonus insularis criados em ovos de Spodoptera frugiperda, em condiçōes de laboratório. Sete Lagoas (Minas Gerais), 1992.

\begin{tabular}{lrrc}
\hline \multirow{2}{*}{$\begin{array}{c}\text { Estágios de } \\
\text { desenvolvimento }\end{array}$} & \multicolumn{3}{c}{ Duração (dias) } \\
\cline { 2 - 4 } & Média & $\mathrm{s}$ & Intervalo de confiança \\
\hline Incubação & 1,80 & 0,42 & $1,63 \leq x \leq 1,97$ \\
Periodo larval & 20,42 & 1,65 & $20,09 \leq x \leq 20,74$ \\
Período pupal & 6,19 & 1,69 & $5,51 \leq x \leq 6,87$ \\
\hline
\end{tabular}

A longevidade média de 10 machos acasalados foi de 8,4 dias, sendo o máximo de 15 e o mínimo de cinco dias. As dez fêmeas acasaladas viveram em 
média 11,6 dias com o máximo de 18 e o mínimo de cinco dias. Esses dados assemelham-se a aqueles obtidos por JACKSON et al. (1978); SWAMIAPPAN \& BALASUbRAmANian (1979) e por POWERS \& OATMAN (1984) para C. blackburni à temperatura média de $33^{\circ} \mathrm{C}$. Segundo esses autores, a longevidade desse parasitóide foi influenciadda pelos hospedeiros e por diferentes temperaturas.

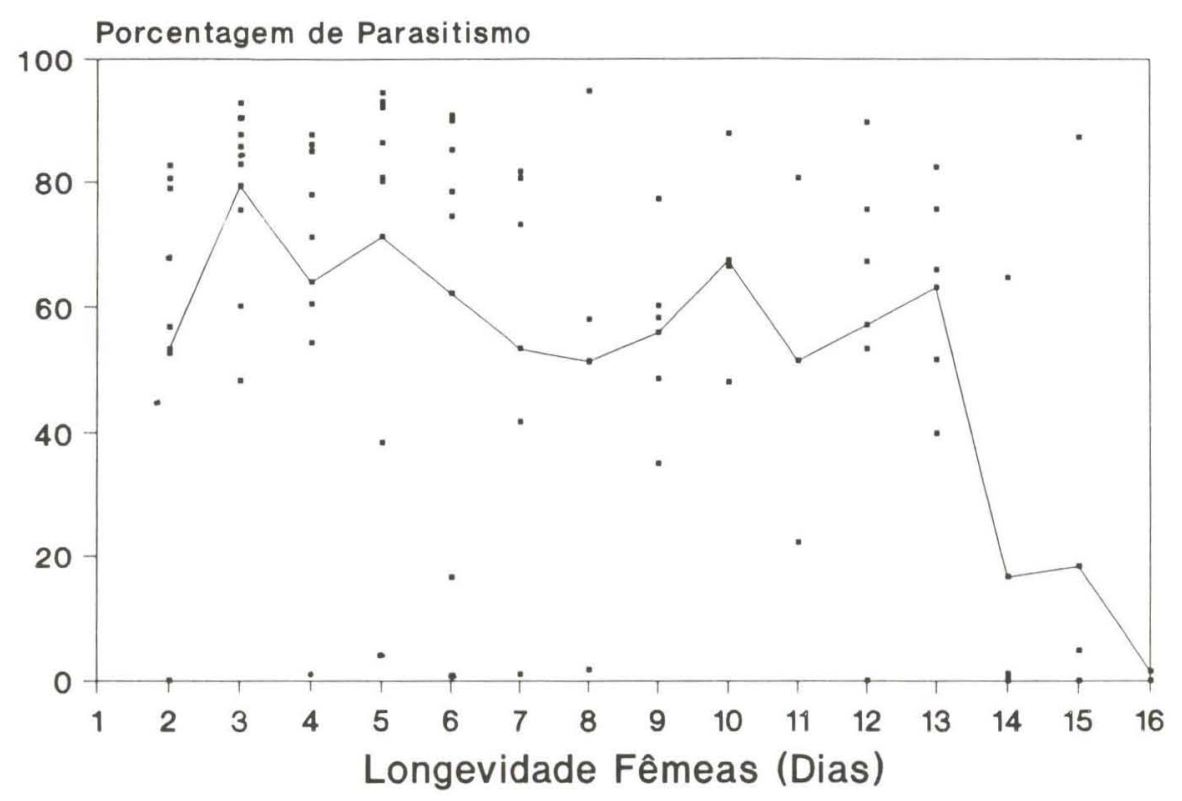

Figura 1. Porcentagem de parasitismo e média diária de ovos de Spodoptera frugiperda, parasitados por fêmea de Chelonus insularis por dia.

O potencial de parasitismo e a longevidade variaram muito de fêmea para fêmea, tendo em média o potencial reduzido em $22 \%$ três dias antes da morte de cada uma. A maior taxa de parasitismo foi verificada quando as fêmeas estavam com três dias de idade, com o máximo de 92,2 e o mínimo de 48,2 ovos parasitados naquele dia (Fig. 1). A média de parasitismo de nove fêmeas de $C$. insularis foi de $60 \%$. Este valor aparentemente baixo comparado aos dados preliminares obtidos pelos autores anteriormente a este trabalho pode ter decorrido da emergência de $80 \%$ de machos. Segundo o Dr. Jeremez McNeil da Université Laval, Quebéc (comunicação pessoal), a criação de parasitóides em laboratório pode requerer a presença de plantas hospedeiras ou fatores como luz incidente para estimular a emergência, além de influenciar na razão sexual dos mesmos, o que não foi considerado na criação de C.insularis durante a condução deste trabalho. Além disso, é possível que a obtenção dos dados de percentual de parasitismo por meio de dissecação dos ovos do hospedeiro forneçam valores mais elevados do que esses 
aqui obtidos. Essa seria uma próxima etapa a ser realizada dentro dessa linha de pesquisa. ASHLEY et al. (1982) verificaram a emergência deste parasitóide em 65 a $80 \%$ das lagartas de $S$. frugiperda.

Em C. kellieae a porcentagem de parasitismo foi de $67,6 \%$ e em $C$. phthorimaeae $90,5 \%$ quando criados em P. operculella (POWERS \& OATMAN 1984). Para C. insularis entre o terceiro e o sexto dia, as fêmeas apresentaram um percentual de parasitismo de 72 a $80 \%$, o que coincide com a fase jovem mais ativa das mesmas (Fig. 1). Ocorreu uma diminuição no sétimo e nono dia com aumentos no $10^{\circ}$ e $13^{\circ}$ dias. A partir do $14^{\circ}$ dia, fêmeas mais velhas parasitaram menor número de ovos e o parasitismo foi cessando, coincidindo com a idade mais avançada das fêmeas. As fêmeas permaneceram parasitando ativamente durante nove dias de vida, após o que houve redução nessa atividade.

\section{REFERÊNCIAS BIBLIOGRÁFICAS}

ASHLey, T.R.; V.H. WAddil; E.R. Mitchell \& J. RYE. 1982. Impact of native parasites on the fall armyworm, Spodoptera frugiperda (Lepidoptera: Noctuidae), in South Florida and release of the exotic parasite Eiphosoma vitticole (Hymenoptera: Ichneumonidae) Environ. Entomol. 11: 833-837.

DE SAntis, L. 1967. Catalogo de los himenopteros Argentinos de la série parasitica incluyendo Bethyloidea. La Plata, Com Invest Cient., 337p.

1980. Catálogo de los himenopteros brasilenõs de la Série Parasítica incluyendo Bethyloidea. Curitiba, Editora da Universidade Federal do Paraná, 395p.

HAFES, M.; M.F.S. TAWFIK \& A. IBRAHIMA. 1980. The imature stages of Chelonus inanitus (L.), a parasite of the cotton leafworm, Spodoptera littoralis (Bolsd), in Egypt Dsich. Ent. Z. N.F. 27: 29-38.

Jackson, C.G.; J.S. Delph \& E.G. Nemmann. 1978. Development, longevity and fecundity of Chelonus blackburni (Hym. Braconidae) as a parasite of Pectinophora gossypiella (Lep. Gelechiidae) Entomophaga 23: 34-35.

Marsh, P.M. 1979. Braconidae. In: K. V. Krombein; P.D. Hu Rd; D. R. SMith \& B.D. BurK (Eds). Catalog of Hymenoptera in America north of Mexico. Washington, Smithsonian Institution Press, v.1, 1198p.

Perkins, W.D.; R. L. Jones; A. N. Sparks; B.R. Wiseman; J.W. SNOW \& W.W. MCMillian. 1973. Artificial diet for mass rearing of corn earworm (Heliothis zea). sl. Agricultural Reserch Service, United States Department of Agriculture, 7p. (Prod. Res. Rep., 154).

Pierce, W.D. \& T.E. Holloway. 1912. Notes on the biology of Chelonus texanus Cress. J. Econ. Entomol. 5: 425-428.

POWERS, N.R. \& E.R. OATMAN. 1984. Biology and temperature responses of Chelonus kelliae and Chelonus phthorimaea (Hymenoptera: Braconidae) and their host, the potato tuberworm, Phthorimaea operculella (Lepidoptera: Gelechiidae). Hilgardia 52: 1-32.

RECHAV, Y. 1978. Biological and ecological studies of the parasitoid Chelonus inanitus (Hym. Braconidae) in Israel. III. Effects of temperature, humidity and 
food on survival of the adult. Entomophaga 23 (1): 89-94.

Swamiappan, M. \& M. Balasubramanian. 1979. Studies on mass multiplication and potentiality of Chelonus blackburni Cam. a braconid parasite of cotton bolloworms. Entomon 5: 73-75.

Ullyett, G.C. 1949. Distribution of progeny by Chelonus texanus Cress. (Hymenoptera: Braconidae). Can. Entomol. 81: 25-44.

Wolcott, G. N. 1948. The insects of Puerto Rico. Jour. Agric. Univ. Puerto Rico 32 (4): 758-759. 